\title{
El capitalismo organizado Entre el orden y el caos
}

\author{
Pablo González Casanova
}

\section{A Imanuel Wallerstein}

\section{PREDICCIÓNY CONSTRUCCIÓN DE}

\section{FUTUROS}

$\mathrm{P}$

or lo general, cuando pensamos en el futuro extrapolamos lo que estamos viviendo. A veces consideramos tendencias más sofisticadas que incluyen los ciclos de distintas dimensiones. En ellos colocamos variables económicas, militares, sociales y políticas. Vaticinamos disminución o aumento en el producto interno bruto (PIB), en las guerras, los suicidios y los crímenes, o fenómenos de crisis y de auge. Llegamos incluso a pensar en términos del fin del sistema o de su continuidad asegurada.

Conforme cambiamos de perspectiva pasamos de pensar en el futuro inmediato y en los próximos años como algo ligado a nuestra propia vida o a la de nuestros hijos y nietos, a pensar en términos de décadas y de los primeros treinta, cincuenta o cien años que vienen. En todos es- tos casos podemos fijarnos en lo que debemos hacer como individuos o colectividades para alcanzar ciertos objetivos vitales en el futuro previsto, o quedarnos como observadores y comentaristas esperando que los hechos se desarrollen de manera más o menos fatal o predeterminada.

A la toma de una posición pasiva contribuyen grandes tradiciones religiosas e ideológicas; algunas muy recientes, como el estructuralismo en que desaparece el sujeto. Esa posición sin sujeto, sin actor, es la menos indicada para comprender un futuro en el que los fenómenos de construcción son tan importantes como quién los construye. Hoy, más que nunca en toda la historia del hombre, la construcción de "futuros conservadores" y "alternativos" por medio de la estructuración y el enfrentamiento de actores y de planes, de sistemas y de escenarios, constituye la esencia de la comprehensión del cambio. Parodiando a Wallerstein, podríamos decir que "la salida dependerá de los detalles de la lucha organizada actual" (Wallerstein y Clesse, 1999: 4-5).

\section{Organized Capitalism: Between Order and Chaos}

PAblo GonZÁlez CaSAnOva: Instituto de Investigaciones Sociales, Universidad Nacional Autónoma de México, México-Distrito Federal. 
En las corrientes de investigación tecnocientífica más avanzadas son riquísimas las aportaciones en relación con los sistemas organizados, con sus contextos y escenarios alternativos. Uno de los avances más importantes es el que mira como una sola unidad a la organización y al caos. A diferencia del concepto clásico de caos, el actual no opone la idea del caos a la del orden establecido, sino que más bien analiza cómo se pasa de la organización al caos y cómo del caos emerge la organización.

Las investigaciones tecnocientíficas sobre las organizaciones y el caos tienen hoy un enorme impacto en la construcción de futuros. Pensaremos poco y mal acerca del futuro inmediato si no pensamos en esas organizaciones y sus relaciones con los fenómenos caóticos actuales y emergentes. Nuestro pensamiento se verá también muy limitado si no consideramos que esas organizaciones determinarán en buena medida los resultados de la lucha en condiciones de turbulencia, inestabilidad, desequilibrio, anarquía de facto. Todo ocurrirá dentro del complejo orden-desorden mundial que vivimos y que, en gran medida, corresponde a la unidad del caos y la organización dentro del modo de dominación y acumulación capitalista que prevalece en el mundo. Pero en medio de la organización-caos mundial, tanto las organizaciones dominantes como las alternativas desempeñarán un papel histórico al que no estamos epistemológicamente acostumbrados. En medio del orden y el caos mundial los llamados "complejos militares-industriales" y "las corporaciones" van a sustituir la "mano invisible del mercado" con la "mano visible" de la organización, como sostuvo Peter Meskins (Meskins, 1998: 153). Los movimientos alternativos tienen que dejar muy clara la importancia de las megaorganizaciones en la historia presente. Ese es el primer punto a destacar.

Una vez aceptada la necesidad de considerar a las organizaciones y al caos desde la perspectiva de las tecnociencias se tiene que pasar al análisis histórico o al estudio de la evolución reciente de las organizaciones en las crisis, las turbulencias, las desestabilizaciones, los desequilibrios, y a la historia de las formas complejas en que las crisis han sido superadas mediante reorganizaciones de las empresas, de los Estados, de los mercados, y mediante reestructuraciones de los sistemas de dominación, apropiación, reproducción ampliada, distribución, exclusión, represión, depredación, parasitismo, explotación. En el estudio se requerirá incluir el análisis de la historia de las mediaciones que permitieron al sistema "cambiar para sobrevivir", y de aquéllas que, en el campo alternativo, trataron de implantar un nuevo sistema y fueron desviadas, cooptadas o eliminadas, aunque lograran éxitos efímeros o parciales, cuyo carácter acumulativo es objeto de conocimiento fundamental.

En todo caso, este segundo fenómeno plantea problemas teóricos que pueden contribuir a esclarecer los límites científicos y técnicos del enfoque anterior y llevarnos a los problemas ético-prácticos de la construcción de una alternativa radical que elimine los rasgos depredadores y autodestructivos de la sociedad actual.

Las limitaciones del estudio tecnocientífico hegemónico acerca de las organizaciones y el caos se advierten con claridad cuando se ve el rechazo cognitivo de los "especialistas" a las categorías articuladas de la dominación y la acumulación de capital. Si las llegan a analizar, desarticulan unas de otras, así como de la categoría de la explotación. Cuando analizan la dominación, por lo general la desligan de la acumulación y de la explotación.

Estas categorías, tabúes para las tecnociencias hegemónicas, han sido, en cambio, profundizadas por las distintas corrientes del pensamiento alternativo socialista, en especial el marxista. Pero en el estudio de la construcción del futuro las nuevas corrientes alternativas no sólo requieren ponerse al día en lo que se refiere a las ciencias dominantes y hacer una autocrítica profunda y creadora de las mismas, sino que, al diseñar una nueva alternativa, no pueden desligar el socialismo de la democracia ni la democracia del socialismo, ni uno y otro de la liberación, como ha ocurrido a menudo durante más de un siglo y medio. En todo caso, los constructores de una alternativa tienen que ver hasta qué punto las tecnociencias de las organizaciones y estructuras dominantes constituyen, a la vez, nuevos obstáculos y nuevos útiles para alcanzar sus objetivos. También necesitan combinar sus objetivos en un complejo concreto para el triunfo de la liberación, la democracia y el socialismo en medio del orden y el caos, que estudian y aplican las "ciencias de la complejidad" de las fuerzas dominantes. Este es el segundo punto a destacar. 
Queremos abordar estos dos puntos viendo primero cómo aparece el futuro de las organizaciones y el caos en los estudios sobre la actual tecnociencia de la guerra, y cómo los estudios sobre los sistemas complejos plantean nuevas posibilidades de estructuración, reflexión y acción a las organizaciones alternativas. El objetivo es conocer el pensamiento actual de los guerreros vinculados a los gerentes, y lo que puede ser relevante para los movimientos alternativos, sistémicos y antisistémicos.

\section{EL CONOCIMIENTO DE LA ORGANIZACIÓN: SU FUNCIONAMIENTOY ESTRATEGIAS}

"Una vez más descubrimos que el modo de hacernos ricos y de hacer la guerra están inextricablemente conectados" (Toffler 1993: 64), dicen los esposos Toffler que, a su modo, dan a conocer "la revolución en el arte de la guerra" para una "revolución en el arte de la paz".

Las grandes compañías están desmantelando —o han desmantelado ya - sus viejas estructuras para establecer "centros de utilidades" (profit centers) o "centros de coordinación y mando" (coordination and managing centers), con gran diversidad de alianzas estratégicas, de "riesgos empresariales compartidos" (joint ventures), de asociaciones y consorcios, muchos transnacionales. Los nuevos "complejos" requieren niveles cada vez más altos de organización del conocimiento y de la información, no sólo en relación con las organizaciones que los integran, sino con la reestructuración de éstas o de algunos de sus componentes y "procesos", tareas propias de la "reingeniería” y las tecnociencias que operan en la producción y los mercados, así como en los escenarios políticos, militares, económicos, sociales y culturales (Toffler y Toffler, 1993: 61-62).

La filosofía de las "respuestas flexibles" en los negocios y en la guerra se practica en complejos integrados por unidades relativamente autónomas. Su filosofía corresponde a lo más avanzado en las prácticas de organizaciones y estrategias (Toffler y Toffler, 1993: 77). El uso "de comandantes de campo" con autonomía de mando se aplica en forma parecida a los "gerentes" periféricos de las unidades trasnacionales y a los "presidentes" asociados de los

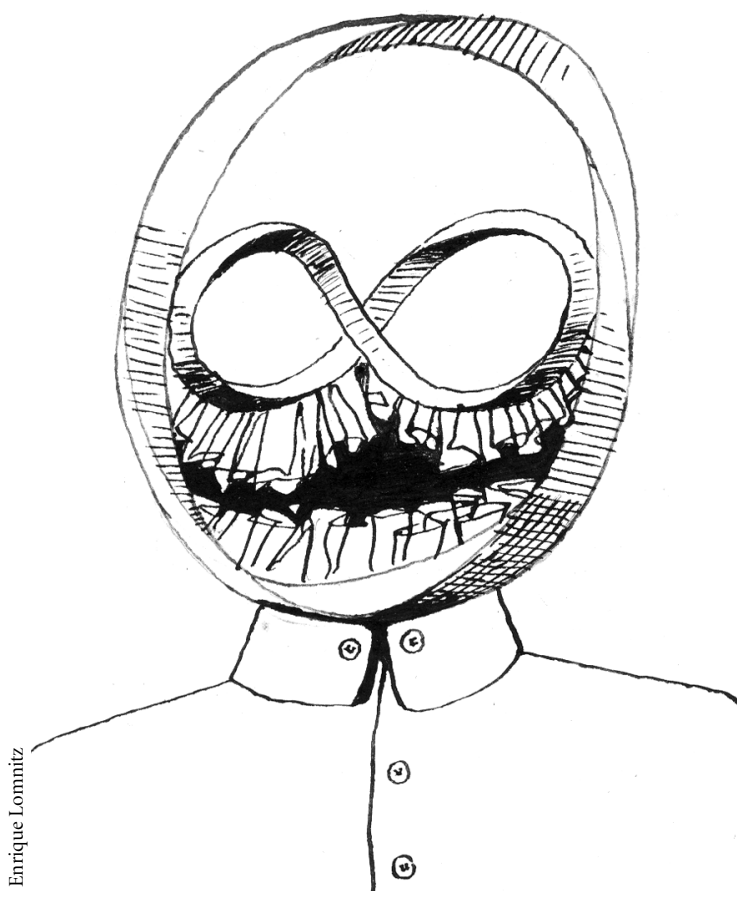

- 167

países dependientes. Dentro de las líneas generales que controla, el mando central deja un margen de libertad más o menos amplio a los titulares de las organizaciones menores. Con éstos comparte la "autoridad de arriba para abajo" (top-down authority) y, entre todos, la revisan y flexibilizan, según los mensajes y conocimientos recibidos desde abajo (Toffler y Toffler, 1993: 77-78).

Los ciber-negocios y las ciber-guerras conocen lo más que pueden del adversario (o competidor), al tiempo que le impiden conocerse y conocer su entorno. La lucha consiste en voltear "el balance de la información y del conocimiento en favor de uno mismo". Al efecto, tanto los complejos militares como "las corporaciones y las compañías" adquieren, procesan, distribuyen y protegen información y conocimiento, negándolos a sus adversarios e, incluso, a sus aliados, o dándoselos en forma selectiva (Toffler y Toffler, 1993: 141-142).

La nueva política de los negocios y de la guerra parte del supuesto de que el conocimiento y la información son inútiles en ciertas manos y cabezas, o cuando se dan a destiempo (in the wrong hands or heads at the wrong time) 
(Toffler y Toffler, 1993: 144). Propone información y conocimiento en las cabezas y las manos apropiadas y en el tiempo adecuado. Conocimiento e información tienen que circular sin demora cuando se les necesita en los centros de reflexión, análisis, educación y publicidad de la "red de compañías" o de "aliados del Tío Sam" (sic) (Toffler y Toffler, 1993: 145-146).

La nueva política se extiende a las redes de la educación en todos sus niveles, desde la primaria hasta el posgrado o la investigación: "Los militares, gerentes y políticos de la 'tercera ola' le dan una importancia masiva (sic) al entrenamiento y la educación en todos los niveles. Sus sistemas para proporcionar 'el entrenamiento adecuado al poder adecuado' son parte del proceso de distribución de conocimiento" (Toffler y Toffler, 1993: 146). En la guerra como en los negocios, "aprender, desaprender y reaprender corresponden a un proceso continuo en todas las categorías políticas" (Toffler y Toffler, 1993: 147).

Los "generales inteligentes" (smart generals) se complementan con los "gerentes inteligentes" (smart managers) y con los "políticos inteligentes" (smart politicians) entrenados en luchas, guerras y pacificaciones, que simulan las batallas en las computadoras antes de librarlas en la realidad.

A la política del conocimiento y la información se suman la del des-conocimiento, el anti-conocimiento y la des-información. Conscientes del valor mercantil, gubernamental y militar del conocimiento, los complejos "empresariales-militares" elaboran estrategias para "la defensa de sus activos de conocimiento (sic) contra cualquier ataque enemigo" (Toffler y Toffler, 1993: 147). Al corriente de los fenómenos complejos no lineales saben que "negar o dar un poco de información o conocimiento puede tener efectos catastróficos" (Toffler y Toffler, 1993: 148). Niegan ese poco de conocimiento o lo dan a conveniencia y a cuentagotas. Y siempre lo consideran como un "activo", una propiedad que comparten con unos y de la que excluyen a otros de acuerdo con los fines de acumulación y dominación del sistema.

El conocimiento y la información de "los complejos" se relacionan también con sus mensajes y acciones de terror (Toffler y Toffler, 1993: 150 ss.), con sus "depredadores virales”, con sus servicios de contrainteligencia y desinformación, de paramilitares y de acciones cívicas. Muchos de sus actos "humanitarios" son paternalistas y caritativos; junto con los de intimidación se proponen dominar la dignidad y la conciencia de los enemigos y convertirlos en sus "servidores" todo lo que se pueda.

Las "estrategias del conocimiento" articulan el pensar, el decir y el hacer de los complejos que hoy gobiernan el mundo. Incluyen una alta precisión en los bombardeos, así como la posibilidad reconocida y el derecho de cometer errores. Aciertos y errores se calculan como formas de sometimiento a lo racional y, también, a lo irracional. Pero, además, forman parte del aprendizaje de las organizaciones dominantes.

El nivel de sofisticación del conocimiento de las organizaciones es muy alto. Leon Roger, director ejecutivo de la "Servicios de Aprendizaje Organizados, 3 M" afirma que éstos tienen como tradición dejar que sus componentes cometan errores. Una vez que los cometen ellos mismos saben que los cometieron y aprenden a no cometerlos de nuevo. Nadie está encima de ellos diciéndoles: “¡Fíjate!” (Champy, 1995: 130). Ellos mismos se fijan.

El conocimiento formal e informal que alcanza cada integrante o miembro de un complejo (o de una compañía) se da en torno a los objetivos comunes del mismo claramente definidos. La enseñanza y el aprendizaje son parte del trabajo, como lo son las pruebas y autopruebas. Los profesores y estudiantes renuevan su compromiso con los valores culturales del complejo, la corporación o la compañía, y ellos mismos los monitorean para ver hasta qué punto se están llevando a la práctica. Dejando "constantes" los valores de la compañía, "la lección más importante que se enseña y que aprenden es que todo cambia" (Champy, 1995: 101-105). No sólo los integrantes del complejo cambian todo para conservar lo principal, sino que están abiertos a todos los cambios para alcanzar los objetivos, intereses y valores del complejo, la corporación o la compañía.

El conocimiento es un "capital intelectual", un activo. La guerra económica o militar es una guerra del conocimiento, la organización y la voluntad. Entre sus promotores mercantiles se encuentran Irujiro Nonalia, Hirotaka Takeuchi y Takeuchi Nokaen, autores de un libro que en inglés se tradujo como The Knowledge-Creating Compa- 
ny (La compañía creadora de conocimiento). Otros son James Brian Quinn, con su Intelligent Enterprise (La empresa inteligente); Tom Stewart, con Intellectual Capital (Capital intelectual); Tom Davenport, con Working Knowledge (El conocimiento empleado) o Karl Sveiby con $\mathrm{Ma}$ naging Know How (El saber del gerente). Con todas las reservas, limitaciones o críticas que suscite el conocimiento del capital y del imperialismo, no se puede ignorar su existencia ni su funcionamiento.

Las corporaciones que producen "conocimiento para la venta" (Knowledge for Sale) han proliferado como asesoras y receptoras de conocimientos, como exportadoras e importadoras y como productoras, pero también como usuarias. La grandeza y efectividad proclamadas por las empresas de punta es que todas sus actividades y discursos se hallan "basados en el conocimiento" (knowledge based) y que descansan en estructuras articuladoras del conocer, el comunicar y el hacer (David y Meyer, 1998: 199-200). La epistemología de las organizaciones tiene "interfases" con la acción de las mismas en la producción y dominación de los mercados y del mundo. Sus estrategias incluyen la teoría y la práctica de un futuro con bifurcaciones y con creación de sorpresas entre el orden y el caos. Constituyen la práctica de lo complejo.

"La planeación de escenarios exige que los gerentes abandonen la idea de una línea a seguir, el supuesto de que sólo existe un futuro determinado que nos concierne. En la planeación de escenarios siempre hay más de un escenario" (Gens, 1997: 44), sostiene Ariede Gens, uno de los autores que escriben libros para gerentes. Quienes diseñan escenarios militares piensan lo mismo. Con unos y otros dominando al mundo entramos al periodo de 2000-2050. Si sus limitaciones para saber lo que realmente pasa son inmensas su conocimiento instrumental no se queda atrás. Esa es la paradoja.

\section{LAS ORGANIZACIONES ALTERNATIVAS COMPLEJAS HACIA EL SIGLO XXI}

Tecnociencias y ciencias de la complejidad se manifiestan con toda claridad en el capitalismo organizado que actúa en medio del orden y del caos. Las tecnociencias y las ciencias de la complejidad contribuyen a la construcción de sistemas autorregulados y adaptativos que se reestructuran en formas interactivas, esto es, que se redefinen a sí mismas y a sus asociados y subordinados, incluso a sus enemigos. La complejidad de las organizaciones del actual sistema capitalista tiene así importantes implicaciones para los movimientos alternativos. Éstos no sólo se ven obligados a reestructurar sus conceptos, sino también la relación entre sus conceptos y sus actos. Innovar sus informaciones y discursos no basta. Los movimientos alternativos tienen que plantearse los conocimientosactos de sus organizaciones con un monitoreo constante para interactuar con éxito frente a las organizaciones complejas dominantes.

Los movimientos alternativos deben saber que las organizaciones dominantes van a redefinirse y a redefinirlos. Tienen que partir del supuesto de que sus propias organizaciones alternativas, sus conceptos-actos, sus informaciones-discursos-acciones, van a dejar de ser como lo son hoy. Ellos también se verán obligados a redefinirse en el curso de los procesos interactivos entre sistemas. Tienen que pensar todo el tiempo en su propia redefinición, y hacerse o rehacerse en formas que les permitan mantener su identidad y actuar mejor en la lucha por la liberación, la democracia y el socialismo.

Los movimientos alternativos deben considerar de forma prioritaria las transformaciones de las organizaciones simples del sistema dominante que se han convertido en verdaderos complejos de corporaciones, empresariales, militares y políticas con redes de mediación, represión y apropiación variables según los espacios centrales y periféricos, y según los periodos históricos "posindustriales", "posmodernos" o "neoliberales", que corresponden al fin del "socialismo real", "del Estado benefactor" $y$ del "Estado populista".

A los ideólogos y dirigentes de los movimientos alternativos no les será fácil aceptar que ellos - y sus herederos- vivirán una etapa de inestabilidad y caos prolongados con desestructuración y reestructuración acentuadas de las organizaciones y los complejos en lucha. Más difícil les resultará entender que las estructuras no lineales "internas" de las organizaciones o complejos y las estructuras no lineales de sus contextos "externos" 


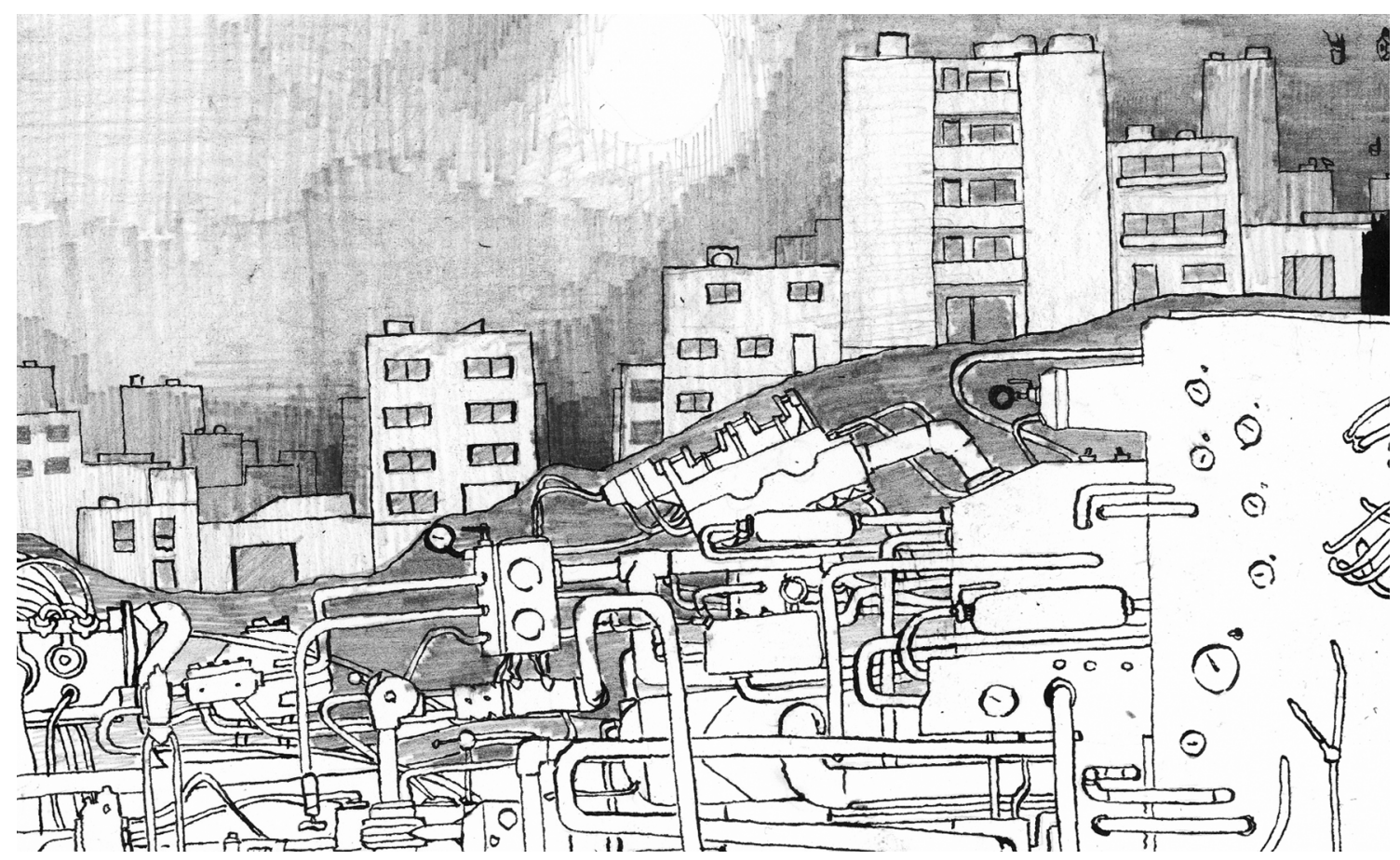

facilitarán o dificultarán las acciones de las organizaciones y complejos en lucha.

Muchos movimientos alternativos - $\mathrm{O}$ sus basesestarán expuestos a "bifurcaciones de bifurcaciones", de las que derivan en fenómenos caóticos y que sólo se pueden enfrentar con redes y fusiones, a sabiendas de que sin redes de unidades articuladas, las unidades desarticuladas son objeto de fácil destrucción.

Los complejos liberadores serán más efectivos si se integran - como los opresores-con unidades o "nodos" autónomos y plurales; y si a más de los vínculos-entre las unidades-autonómas establecen jerarquías con las ramas "centrales" y disciplinadas de "seguridad alternativa" en lucha contra la dominación, la mediación, la represión y la apropiación excluyente. Las distintas combinaciones serán determinantes para el desenlace de los procesos y podrán plantear escenarios en los que primero se acaben los complejos industriales dominantes antes que el mundo, o en que eventualmente se negocie un cambio histórico para que el mundo no se acabe. Estos escenarios no se pueden descartar.
La construcción de una estrategia alternativa implica cuestionar nuestra forma de pensar en sistemas simples. Por sentido común estamos acostumbrados a pensar y actuar con formas de razonamiento que corresponden a sistemas simples. No es cosa de descartar siempre, y en todas las circunstancias, esas formas de pensar, predecir, actuar, organizar y luchar. Hay fenómenos lineales que siguen siendo muy significativos incluso en tiempos y espacios amplios; funciones, interacciones o relaciones sociales que, en ciertos momentos y circunstancias - como las crisis-, ocupan un segundo plano, pero que tienden a reaparecer aunque sea bajo nuevas formas. No podemos descartar organizaciones centralizadas con líneas jerárquicas funcionales para muchos fines de resistencia y sobrevivencia; ni impedir que en los procesos de descentralización circule toda la información necesaria en todas las redes y nódulos de un sistema autorregulado. Pero a nuestro conocimiento acumulado y a nuestro pensamiento crítico necesitamos agregar el monitoreo de las informaciones y análisis sobre fenómenos emergentes en que varían conceptos, opciones y acciones de una manera 
creadora y novedosa, a veces sorprendente, como ocurre a lo largo del proceso de la Revolución cubana iniciada en 1959, o del movimiento zapatista, que emergió en el México de 1994. Registrar los conceptos-actos, las informaciones de lo que se hace, las narrativas y discursos vinculados a acciones y proyectos de acción a fin de desentrañar lo que cambian y conservan, así como sus efectos inmediatos y laterales, previstos e imprevistos, esperados y sorpresivos, es una tarea fundamental para pensar, hablar (o escuchar), proponer y actuar en sistemas complejos.

Frente a los sistemas lineales en que los comportamientos son fáciles de deducir y predecir, los sistemas complejos generan bifurcaciones a las que los movimientos alternativos tienen que estar atentos; o efectos desproporcionados en que una pequeña acción se multiplica por encima de cualquier experiencia anterior; o redefiniciones en las que las organizaciones y redes alternativas después de una batalla ya no son las mismas de antes, como tampoco lo son las que dominan el sistema. En la segunda batalla, unas y otras han cambiado. Al reajuste de la conducta de cada parte por las experiencias - $\mathrm{O}$ como retroalimentación (feadback) — se añaden las variaciones en las espectativas de sus integrantes individuales y colectivos y en la estructura de las conductas para enfrentar desenlaces futuros (feadforward). En éstos no sólo se consideran los "efectos laterales positivos" para uno, sino los "daños colaterales" que se pueden infligir al enemigo.

Al pensar en un sistema complejo como el capitalismo organizado es necesario pensar en dos o tres sistemas más auto-regulados y contradictorios. Siempre dos o más sistemas luchan o se entienden, oprimen o se liberan, se disocian o se asocian. El concepto de un sistema único, por significativo que sea, no es el de un sistema complejo. Tampoco el de un sistema dialéctico. El concepto de un sistema único en el capitalismo organizado es fuente de errores elementales frecuentemente ocultados por un juego de espejos conocido como "enajenación" o "cosificación" del otro y de sus posibilidades de reestructuración autorregulada, y como racionalización y ensalzamiento del sistema en que uno domina o desde el que uno lucha para que domine. En el primer caso, a los demás sistemas sólo se les considera como "con- texto"; en el segundo no se piensa siempre en la capacidad que tienen los sistemas dominantes de rehacerse, de redefinirse sino hasta que ya se rehicieron o redefinieron, como ocurrió a fines del siglo XIX con el advenimiento del capitalismo monopólico y el imperialismo.

Tampoco es posible ignorar a otros actores que, dentro o fuera de un sistema, se pueden sumar abierta o intensamente a la lucha generando crisis mayores de las previstas, crisis exponenciales. Tomar en cuenta sólo a los actores originales de un conflicto o un consenso y extrapolar su lucha o su cooperación al futuro es no comprender los sistemas complejos, llenos de mediaciones y mucho más capaces de "absorber choques" de lo que originalmente se pensó. Frente a las organizaciones simples a que estamos acostumbrados, con centralización piramidal de decisiones, es necesario pensar siempre en términos de organizaciones de corporaciones y de complejos que combinan las redes con autonomías y jerarquías. Las organizaciones alternativas no se van a distinguir por mayores autonomías y menores jerarquías, sino por la mayor participación de sus integrantes en la redefinición de unas y otras.

En las organizaciones complejas del sistema dominante se procura no desvincular los conceptos de los actos, ni unos y otros de la información y los discursos. Se busca articular de manera funcional al sistema, conceptos, discusiones, informaciones y actos. Se propone revisar constantemente su "funcionamiento" para controlar mejor las "fluctuaciones" imprevistas de actores y contextos interactivos. Las estructuraciones y reestructuraciones de sistemas de actores y contextos interactivos son motivo de un estudio sistemático sobre "la sorpresa" y sobre la planeación informada y que se informa. Los movimientos y organizaciones alternativos tienen que adoptar un comportamiento semejante, que incluya el análisis de disfunciones y contradicciones.

Frente a la débil interacción de los componentes de muchas organizaciones alternativas — característica de los sistemas simples-, frente a sus sub-culturas y sectarismos, se impone así la necesidad de construir pluralismos culturales, religiosos e ideológicos, así como articulaciones de conceptos-informaciones-discursos-actos de interés común, todos ellos creadores de sistemas complejos, 
de alianzas, frentes, redes, tanto en los "centros" como en las "periferias".

Que esas alianzas, frentes y redes no abandonen el referente de las clases y busquen la hegemonía de los trabajadores es un objetivo a ser precisado con las definiciones y redefiniciones de los pueblos, los ciudadanos y los propios trabajadores en lucha por la democracia, la liberación, el socialismo. El legado de los movimientos revolucionarios, de sus contradicciones internas de clase y de la consolidación del proyecto liberador, democrático y socialista — en el que Cuba destaca a nivel mundial— debe ser motivo de una reflexión también mundial, de carácter prioritario, que combine la narrativa con la historia y la reestructuración de los movimientos revolucionarios y radicales más exitosos, así como de los que se perdieron en el camino.

Los sistemas de redes autónomas combinados con subsistemas de mandos centrales y de centros coordinadores tendrán en los movimientos alternativos mayor importancia que los sistemas de "partidos" y organizaciones relativamente homogéneos propios de los sistemas simples, y que las coaliciones homogeneizadas por una clase, una ideología o un partido. Plantearán problemas de creación histórica en la cultura, la política, la economía y la sociedad de "un mundo hecho de muchos mundos". Plantearán la democracia plural —respetuosa de razas, religiones y filosofías-, a la vez participativa y representativa, como la ideología más funcional para un proyecto mundial alternativo. Su éxito dependerá, en gran medida, de la redefinición incluyente de las comunidades científicas y humanísticas, críticas, alternativas y revolucionarias de los distintos países del mundo, en tanto asuman como un problema epistemológico, articulador, la ética como poder, pues no se trata sólo de prever el futuro, sino de construirlo y de luchar por su construcción.

En tiempos recientes, conceptos como orden y caos, determinismo e impredictibilidad, incertidumbre y bifurcaciones han sido separados excesivamente de los que se refieren a los sistemas auto-regulados, adaptativos y auto- poiéticos (Maturana y Varela, 1980; González Casanova, 2004). Aunque unos y otros están envueltos en contradicciones, los sistemas autorregulados se redefinen a sí mismos y redefinen su contexto para seguir dominando y explotando los recursos naturales y humanos (Wallerstein, 1999).

En el actual capitalismo corporativo los movimientos sociales antisistémicos necesitan dominar el nuevo planteamiento de los sistemas complejos que dominan el mundo entre el orden y el caos. Con Henri Lefebvre tienen que recordar "la capacidad de recuperación del capitalismo" y "la posibilidad de catástrofes" irremediables (Lefèbvre, 1972: 191) si no se construye un mundo alternativo.

\section{Bibliografía}

Champy, James, 1995, Reengeneering Managment, Harper Bussiness, Nueva York.

David, Stan y Christopher Meyer, 1988, Blur the Speed of Change in the Connected Economy, Warner, Nueva York.

Gens, Ariede, 1997, The Living Company Boston, Harvard Business School Press, Harvard.

González Casanova, Pablo, 2004, Las nuevas ciencias y las humanidades. De la academia a la política, Anthropos, Barcelona.

Lefèbvre, Henri, 1972, Le droit à la ville suivi de Espace et politique, Anthropos, París.

Maturana, J. y F. Varela, 1980, Autopoiesis and Cognition. The Realization of the Living, Reidel, Dordrecht.

Meskins, Peter, 1998, "Work, New Technology and Capitalism", en Robert W. McChesney et al., Capitalism and the Age of Information, Monthly Review, Nueva York.

Toffler, Alvin y Heidi Toffler, 1993, War and Anti-War. Survival at the Dawn of the 21st Century, Little Brown, Boston.

Wallerstein, Immanuel, 1999, The End of the World As We Know It. Social Science for the Twenty-First Century. University of Minnesota Press, Minneapolis y Londres.

— y Armand Clesse (eds.), 1999, The World We Are Entering, 2000-2050, Luxembourg Institute for European and International Studies, Luxemburgo. 